\title{
Lignification in developing culms of bamboo Sinobambusa tootsik
}

\author{
Taku Tsuyama $^{1} \cdot$ Natsumi Shimada $^{1} \cdot$ Taichi Motoda $^{1} \cdot$ Yasuyuki Matsushita $^{2} \cdot$ \\ Yoshio Kijidani $^{1} \cdot$ Kazuhiko Fukushima $^{2} \cdot$ Ichiro Kamei $^{1}$
}

Received: 25 March 2017/Accepted: 29 June 2017/Published online: 9 August 2017

(C) The Japan Wood Research Society 2017

\begin{abstract}
Bamboos are among the largest woody grasses and grow very rapidly. Although lignin is a crucial factor for the utilization of bamboo biomass, the lignification mechanism of bamboo shoots is poorly understood. We studied lignification in the bamboo Sinobambusa tootsik during culm development. Elongation growth began in May and ended in late-June, when the lignin content was approximately half that in mature culms. Thioacidolysis analysis indicated that $p$-hydroxyphenyl units in lignin formed even at late stages of lignification. The syringyl/ guaiacyl ratio varied during culm development. Various lignin precursors were detected in developing culms by liquid chromatography-mass spectrometry. The ferulic acid content decreased from May to June, indicating that ferulic acid was utilized in early stages of cell wall formation. Monolignol glucosides were detected at early stages of lignification, whereas the contents of monolignols, coniferaldehyde, sinapaldehyde, $p$-coumaric acid, and ferulic acid peaked at later stages of lignification. Therefore, lignin precursors may be supplied differentially during the lignification process. In August, the rate of lignification decreased, although the contents of various lignin precursors peaked, implying that the rate-limiting
\end{abstract}

Part of this article has been presented at the 67th Annual Meeting of the Japan Wood Research Society, March 2017, Fukuoka, Japan.

Ichiro Kamei

kamei@cc.miyazaki-u.ac.jp

1 Division of Forest Science, Department of Forest and Environmental Sciences, Faculty of Agriculture, University of Miyazaki, 1-1 Gakuenkibanadai-nishi, Miyazaki 889-2192, Japan

2 Graduate School of Bioagricultural Sciences, Nagoya University, Nagoya 464-8601, Japan step in the cessation of lignification in bamboo is transport or polymerization of lignin precursors, rather than their biosynthesis.

Keywords Bamboo $\cdot$ Development $\cdot p$-Hydroxyphenyl unit $\cdot$ Lignin precursor $\cdot$ Transport

\section{Introduction}

Bamboos are among the largest woody grasses and typically show a rapid growth rate. Utilization of bamboo biomass is an increasingly important research focus [1-5]. Information on the content and distribution of lignin at each developmental stage is crucial for the exploitation of bamboo biomass [6-8]. However, the mechanism of lignification in bamboo shoots remains poorly understood.

The lignification process involves three steps: biosynthesis, transport, and polymerization of lignin precursors. Enzymes involved in the biosynthesis or polymerization of lignin precursors have been investigated in bamboo as well as gymnosperm and angiosperm trees [9-11]. A few studies have explored the transport mechanisms of lignin precursors [12-14].

A knowledge of the distribution of lignin and lignin precursors may provide insights into the lignification mechanism, including the transport step. Several studies have elucidated the distribution of lignin and coniferin, the glucoside of coniferyl alcohol, in differentiating xylem of gymnosperm [15-17]. Lignification and the occurrence of lignin precursors have been examined in the angiosperms Magnolia kobus [18] and poplar [16]. The results of those studies suggested that the lignin precursors are coniferin in gymnosperms and M. kobus, and sinapyl alcohol in poplar. 
In bamboo, lignin distribution at the subcellular level has been investigated using electron microscopy and ultraviolet microscopy [19]. In the previous studies, lignification during growth was studied at the cellular level by Wiesner staining of different internodes of the culm [20, 21]. Lignin content during elongation growth was examined at several heights in shoots of the bamboos Phyllostachys pubescens [22] and Phyllostachys bambusoides [8]. However, structural changes in lignin and the occurrence and distribution of lignin precursors in bamboo culms have not been elucidated.

In this study, we investigated the development and lignification of culms of the bamboo Sinobambusa tootsik. We also determined the distribution of lignin precursors, including $p$-coumaric acid and ferulic acid, in developing culms. These findings provide novel insights into the lignification mechanism in bamboo.

\section{Materials and methods}

\section{Chemicals}

Coniferin and syringin were donated by Professor Emeritus N. Terashima, Nagoya University, Japan. Other chemicals, except for $p$-coumaryl alcohol and $p$-glucocoumaryl alcohol, were purchased from Wako Pure Chemical Industries (Osaka, Japan), Tokyo Chemical Industry Co. (Tokyo, Japan), or Sigma-Aldrich (St Louis, MO, USA).

\section{Syntheses of $p$-glucocoumaryl alcohol and $p$ - coumaryl alcohol}

p-Glucocoumaryl alcohol was synthesized as described previously [23]. Synthesis of $p$-coumaryl alcohol basically followed $p$-glucocoumaryl alcohol synthesis with some modifications. Briefly, a mixture of $p$-hydroxybenzaldehyde $(6.0 \mathrm{~g})$, monoethyl malonate $(8.4 \mathrm{~g})$, pyridine $(6 \mathrm{~mL})$, and piperidine (10 drops) was heated at $60{ }^{\circ} \mathrm{C}$ for $24 \mathrm{~h}$. After acidification ( $\mathrm{pH} 3$ ), the reaction mixture was extracted with ethyl acetate followed by extraction with saturated sodium hydrogen carbonate solution and with $10 \%$ sodium hydrogen sulfite. The organic layer was washed with water, and then concentrated. Recrystallization from the mixture with ethyl acetate and hexane yielded $p$-coumaric acid ethyl ester (5.6 g, yield 59\%).

Lithium aluminum hydride $(0.8 \mathrm{~g})$ was placed in a flask and absolute tetrahydrofuran $(50 \mathrm{~mL})$ was added. $p$-Coumaric acid ethyl ester solution ( $1.0 \mathrm{~g}$ in $15 \mathrm{~mL}$ of absolute tetrahydrofuran) was added drop-wise over $1 \mathrm{~h}$ with stirring, and the reaction was maintained for $2 \mathrm{~h}$. After acidification $(\mathrm{pH} 3)$, the reaction mixture was extracted three times with ethyl acetate. The organic layer was washed with brine and then removed to obtain $p$-coumaryl alcohol $(0.12 \mathrm{~g}$, yield $15 \%)$.

\section{Extraction of lignin precursors}

The S. tootsik plants used in these experiments were growing at the University of Miyazaki campus. The samples were collected between May and September (see Table 1), and in November (Fig. 1c). Three culms of bamboo with a similar total height were collected at each sampling. Culms that were more than 1 year old were collected as "mature" samples, although their exact age was unknown. The lowest internode above the ground or the rooted internode was designated as 0 , and internodes were numbered from the bottom to the top of the culm. Blocks containing both the outer- and inner-most cells of the internode were excised from the top portion of the 2nd, 4 th, 6th, 8th, and 10th internodes. The blocks were frozen in liquid nitrogen and stored at $-80{ }^{\circ} \mathrm{C}$ until use in the following experiments.

Transverse cryosections were obtained from each frozen specimen block and placed in a $1.5-\mathrm{mL}$ vial at $-20{ }^{\circ} \mathrm{C}$. The vials were weighed before and after adding the sections to determine the weight of fresh sections. After weighing the vial, $1-\mathrm{mL} 80 \%$ methanol was added and the sections were extracted overnight at $4{ }^{\circ} \mathrm{C}$. The extract was collected and lignin precursors were determined by liquid chromatography-mass spectrometry (LC-MS). The sections were treated with acetone overnight after the methanol extraction. After discarding the supernatant, the sections were dried and used for lignin analyses.

\section{Quantification of lignin}

The thioglycolic acid (TGA) method [24] was used to quantify lignin. Briefly, samples were reacted with 1-mL $3 \mathrm{~N}$ $\mathrm{HCl}$ and $0.1-\mathrm{mL}$ TGA in 2-mL tubes with screw caps at $80{ }^{\circ} \mathrm{C}$ for $3 \mathrm{~h}$. The tubes were centrifuged at $20,000 \times g$ for $10 \mathrm{~min}$, the pellet was washed with $1-\mathrm{mL}$ distilled water, then 1-mL $1 \mathrm{~N} \mathrm{NaOH}$ was added, and the tube was shaken vertically at $80 \mathrm{rpm}$ overnight. After centrifugation as described above, 1-mL supernatant was mixed with $0.2-\mathrm{mL}$ concentrated $\mathrm{HCl}$. The TGA lignin was precipitated at $4{ }^{\circ} \mathrm{C}$ for $4 \mathrm{~h}$ and collected by centrifugation as described above. The pellet was dissolved in $1 \mathrm{~N} \mathrm{NaOH}$ and absorption at $280 \mathrm{~nm}$ was measured to determine the amount of TGA derivatives from a standard curve prepared using known concentrations of bamboo milled-wood lignin [24].

\section{Characterization of lignin}

Extractive-free samples were used to characterize lignin using the microscale thioacidolysis method [25]. 
Table 1 Samples used in the present study

\begin{tabular}{llll}
\hline Sample name & Sampling date & Total culm length $(\mathrm{m})$ & 1st-10th internodes length $(\mathrm{m})$ \\
\hline M-May & 14 and 18 May 2016 & $0.65 \pm 0.01$ & $0.34 \pm 0.05$ \\
L-May & 27 May 2016 & $2.94 \pm 0.09$ & $2.42 \pm 0.10$ \\
M-Jun & 8 Jun 2016 & $7.66 \pm 0.53$ & $5.85 \pm 0.24$ \\
L-Jun & 22 Jun 2016 & $12.40 \pm 1.16$ & $5.78 \pm 0.14$ \\
Jul & 13 Jul 2016 & $12.52 \pm 0.61$ & $6.08 \pm 0.46$ \\
Aug & 12 Aug 2016 & $13.77 \pm 0.97$ & $6.27 \pm 0.25$ \\
Sep & 9 Sep 2016 & $12.39 \pm 0.45$ & $5.89 \pm 0.32$ \\
Mature & 12 Aug 2016 & $12.75 \pm 1.26$ & $5.40 \pm 0.26$
\end{tabular}

To obtain samples at a uniform developmental stage in each sampling, culms of as similar total length as possible were collected. 'Mature' samples were culms that were older than 1 year old. Length data are mean $\pm \mathrm{SD}$ of three biological replicates
Tetracosane was used as the internal standard. Gas chromatographic-mass spectrometric (GC-MS) analyses were performed on a GCMS-QP 2010 SE instrument (Shimadzu, Kyoto, Japan) equipped with an Inert Cap column (60 m × $0.25 \mathrm{~mm}$ i.d.; GL Sciences, Tokyo, Japan). The sample volume injection was $1 \mu \mathrm{L}$ in the pulsed splitless mode. The injector and transfer line were operated at 250 and $280{ }^{\circ} \mathrm{C}$, respectively. The initial oven temperature was $200{ }^{\circ} \mathrm{C}$ (held for $1 \mathrm{~min}$ ) and increased at $4{ }^{\circ} \mathrm{C} \min ^{-1}$ to $280{ }^{\circ} \mathrm{C}$ and held for $10 \mathrm{~min}$. Quantitative evaluation of $p$ hydroxyphenyl (H), guaiacyl (G), and syringyl (S) monomers was conducted using ion chromatograms reconstructed from their prominent benzylic ions at $\mathrm{m} /$ z 239, 269, and 299, respectively.

\section{LC-MS analysis}

The methanol extract stored at $-80{ }^{\circ} \mathrm{C}$ was centrifuged at $20,000 \times g$ for $20 \mathrm{~min}$, and then, the supernatant was filtered through a membrane filter $(0.45 \mu \mathrm{m})$ before LC-MS analysis. The LC-MS analysis was carried out using a Dionex Ultimate 3000 UHPLC system (Thermo Scientific, Waltham, MA, USA) connected to a Q-Exactive mass spectrometer (Thermo Fisher, Rockford, IL, USA). A 2- $\mu \mathrm{L}$ aliquot of each sample was injected onto a reversed-phase InertSustain C18 column $(100 \mathrm{~mm} \times 2.1 \mathrm{~mm}$ i.d., $2 \mu \mathrm{m}$; GL Sciences, Tokyo, Japan) controlled at $35{ }^{\circ} \mathrm{C}$. The flow rate was $0.3 \mathrm{~mL} \mathrm{~min}^{-1}$, and the gradient program was as follows: $15 \%(\mathrm{v} / \mathrm{v})$ methanol held for $5 \mathrm{~min}$, increased to $35 \%$ methanol at $15 \mathrm{~min}$, increased to $80 \%$ methanol at $20 \mathrm{~min}$, and held until $28 \mathrm{~min}$ with constant $0.1 \%$ acetic acid. The mass spectral data were collected in positive or negative ion polarity using a quadrupole mass spectrometer equipped with a heated capillary electrospray interface. Lignin precursors were identified and determined by comparisons with standard curves calculated from MS data for authentic compounds. The retention time and mass of each authentic compound (mass tolerance $=3 \mathrm{ppm}$ ) used for identification were as follows: p-coumaryl alcohol (10.1 min,,$+ m / z$ 133.065), $p$-glucocoumaryl alcohol (4.1 min,,$+ \mathrm{m} / \mathrm{z} 335.110$ ), coniferaldehyde (15.5 min, -, $m / z$ 177.056), coniferyl alcohol (12.3 min,,$+ \mathrm{m} /$ z 163.075), coniferin (6.2 $\mathrm{min},+, \mathrm{m} / \mathrm{z} 365.121)$, sinapaldehyde (16.4 min,,$- \mathrm{m} / \mathrm{z}$ 207.066), sinapyl alcohol (13.3 $\mathrm{min},+, \mathrm{m} / \mathrm{z}$ 233.078), syringin (8.7 min,,$+ \mathrm{m} /$ z 395.131), $p$-coumaric acid (13.2 min,,$- \mathrm{m} / \mathrm{z}$ 163.040), and ferulic acid (15.0 $\mathrm{min},-, \mathrm{m} / \mathrm{z}$ 193.050).

\section{Results and discussion}

\section{Lignification and elongation growth of $S$. tootsik shoots}

Shoots of $S$. tootsik began growing in mid-May, and finished elongating at the end of June, by which time the shoots were about 13-m high (Fig. 1a). The lowest measured internode (2nd) finished growing earlier and attained a shorter length than did the other internodes (Fig. 1b). The internode length on mature culms gradually increased towards the center of the culm (Fig. 1c), as reported for other bamboo species [22, 26]. Given that $S$. tootsik has comparatively long and few internodes, the 10th internode was at approximately the longitudinal center of the culm (Table 1; Fig. 1c). The leaf sheaths had entirely abscised in July. Foliation of $S$. tootsik shoots occurred in late October.

In the early stage of culm development (May and June), the lignin content was higher in the lower internodes than in the higher ones (Fig. 2a), as reported previously [20, 22]. After elongation finished at the end of June, the lignin contents became almost identical among all the analyzed internodes. At the end of June, when culms had finished elongating, the lignin content was approximately half that in mature samples. Lignification in S. tootsik shoots appeared to cease during August. The lignification rate (change in lignin content per day, $\% \mathrm{~d}^{-1}$; Fig. 2b) 

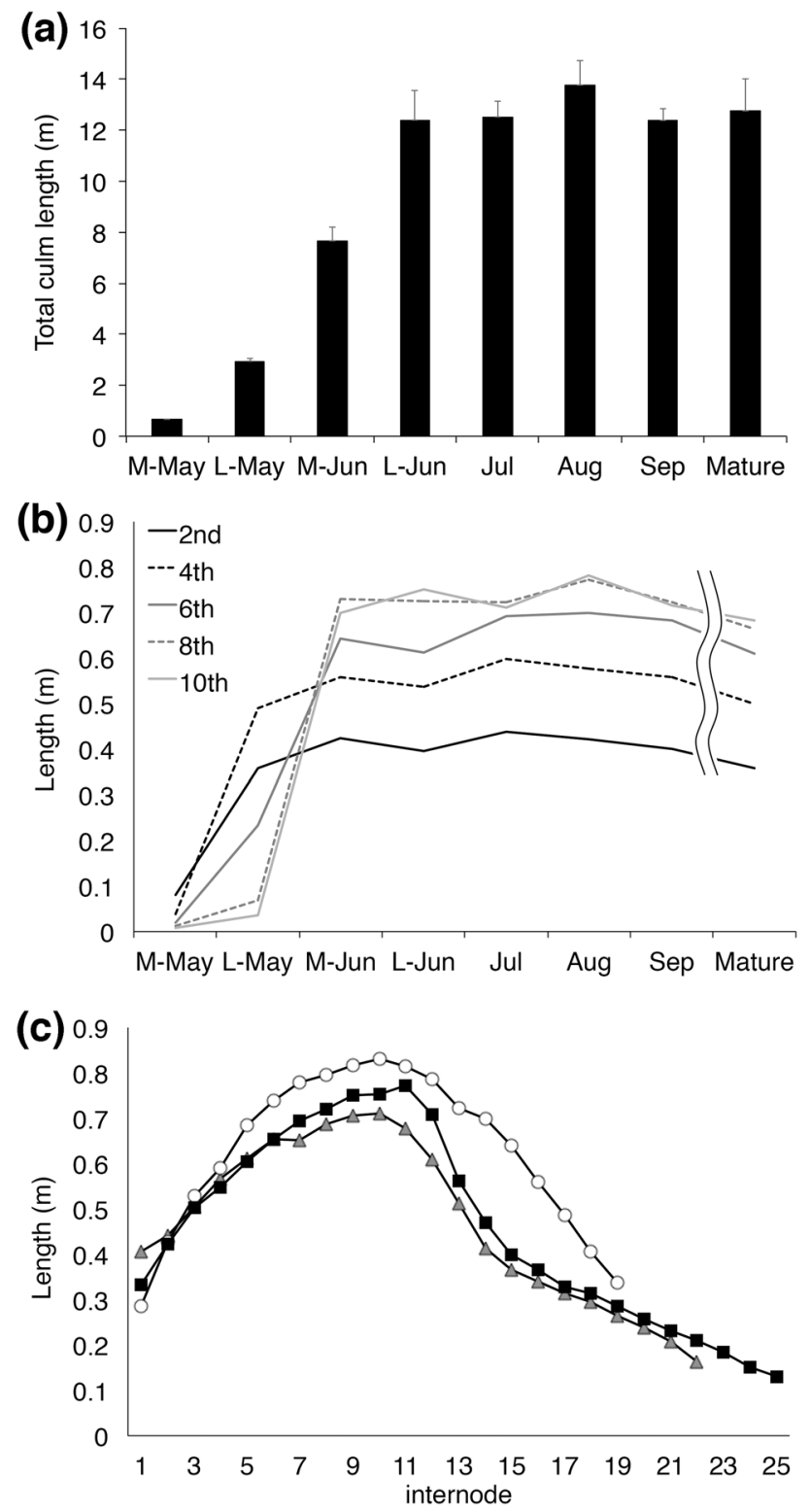

Fig. 1 Elongation growth of Sinobambusa tootsik culms. a Total length of culm samples (see Table 1). Data are mean \pm SD of three biological replicates. b Length of 2nd, 4th, 6th, 8th, and 10th internodes of culms (see Table 1). Data are means of three biological replicates. c Length of each internode of mature ( $>1$ year old) culms collected in November

gradually decreased in August and September. At 4 months after shoot initiation, the lignin content of culms was approximately $80 \%$ of that in mature samples (Fig. 2a). In Phyllostachys heterocycla, lignification was completed within one growing season as determined by the Klason method [20]. In another study, microspectrophotometric analyses suggested that lignification of fiber and parenchyma cell walls continued for up to 7 years [21]. Other studies have indicated that cell wall thickening may occur in 12-year-old culms [27]. Thus, cell wall formation and
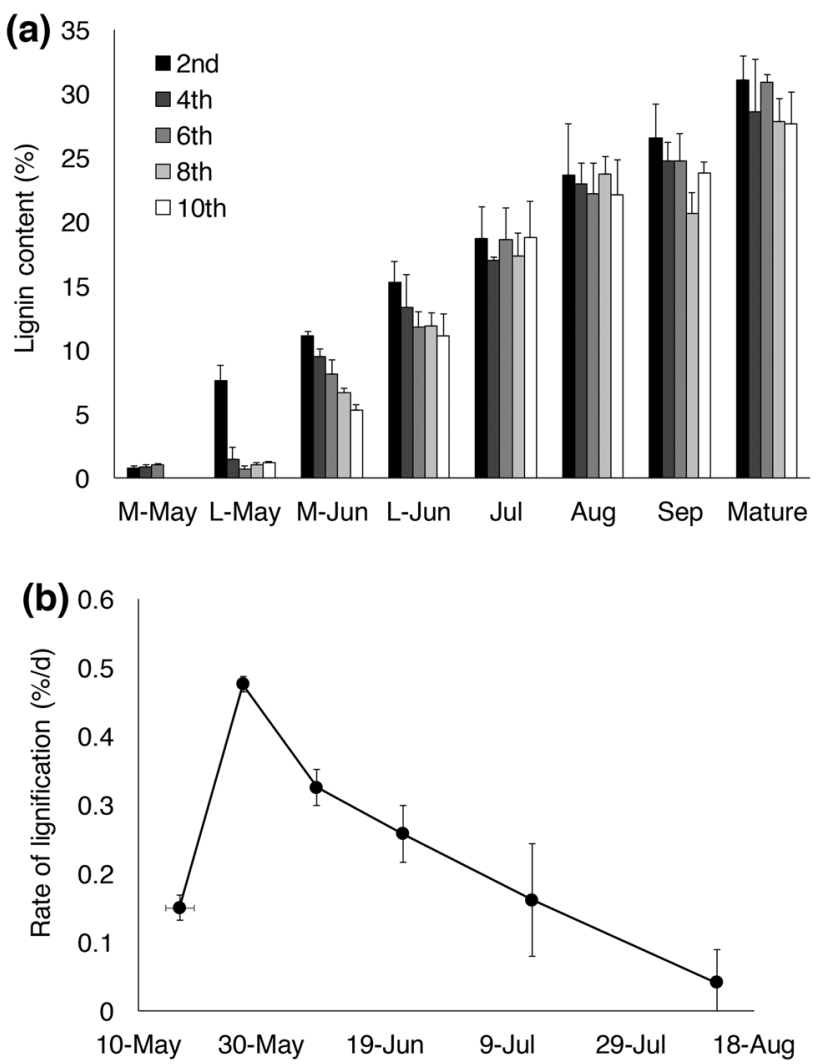

Fig. 2 a Distribution of lignin in developing culms of S. tootsik. Lignin content was determined by thioglycolic acid method [24]. See Table 1 for sampling details. b Rate of lignification in S. tootsik culms. Lignification rate $\left(\% \mathrm{~d}^{-1}\right)$ was calculated as follows: $v_{n}=\left(C_{n+1}-C_{n}\right) /\left(D_{n+1}-D_{n}\right)$, where $v_{n}$ is lignification rate of $n$th sampling, $C_{n}$ is average lignin content (\%) of all internodes investigated at $n$th sampling, and $D_{n}$ is data of $n$th sampling. Data were plotted as $(x, y)=\left(v_{n}, D_{n}\right)$. Data are mean $\pm \mathrm{SD}$ of three biological replicates

lignification in bamboo may continue incrementally over several years.

\section{Lignin structure in developing shoots of $S$. tootsik}

Although several studies have investigated lignification in bamboo, little is known about lignin structure in developing bamboo. Thioacidolysis, which specifically cleaves $\beta$ $O-4$ linkages, can reveal aspects of lignin structure in the cell wall. Lignin in shoots of $S$. tootsik had fewer $\beta-O-4$ linkages in the early stage of lignification, as indicated by yields of thioacidolysis-releasable monomers (Fig. 3). The ratio of thioacidolysis-releasable H:G:S units in lignin of mature culms was 5:41:55.

Heterogeneous formation of lignin occurs in the differentiating xylem of trees [28, 29]; the $\mathrm{H}$ monomer is mainly incorporated into the lignin polymer during the early stages of lignification, followed by the incorporation of substantial amounts of $\mathrm{G}$ units, and subsequently the incorporation 


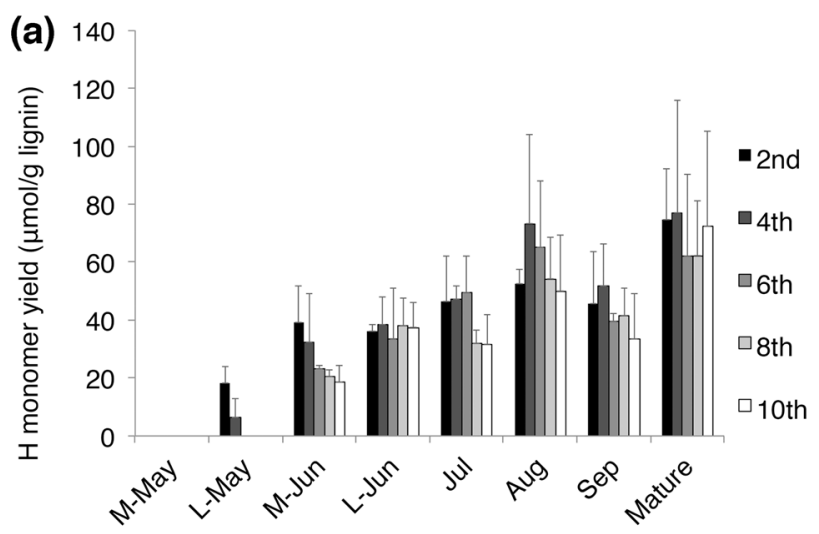

(b) 1200

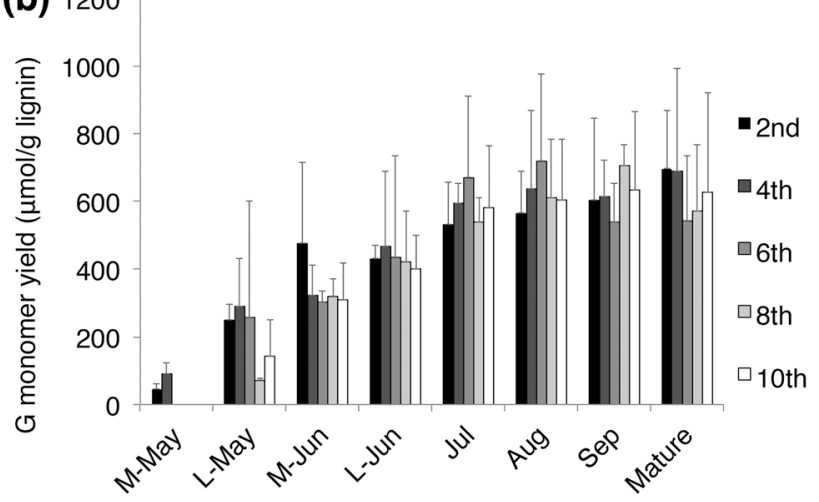

(c) 1600

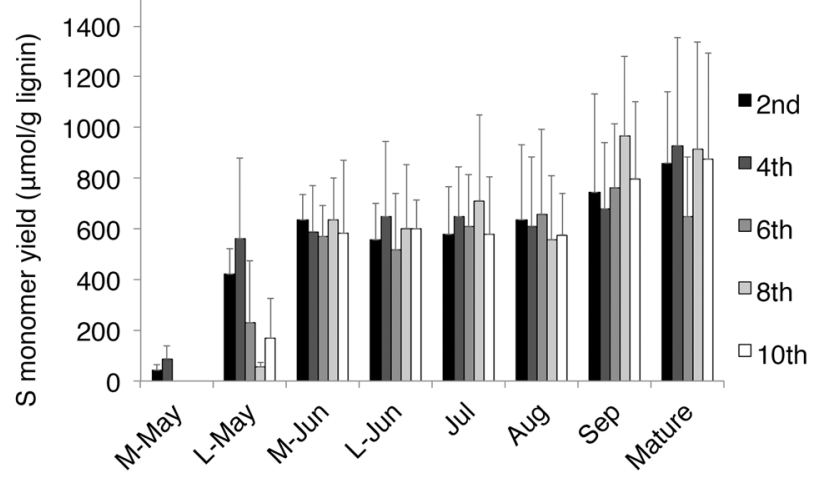

Fig. 3 Thioacidolysis yields from developing culms of S. tootsik. Yield of thioacidolysis-releasable monomers derived from a $p$ hydroxyphenyl $(\mathrm{H})$ units, b guaiacyl $(\mathrm{G})$ units, and c syringyl (S) units. H units were not detected in mid-May sample or in 6th, 8th, and 10th internodes of late-May sample. See Table 1 for sampling details. Data are mean \pm SD of three biological replicates

of S units. In shoots of S. tootsik, however, thioacidolysisreleasable yields of $\mathrm{H}$ monomers continued to increase even in August, during the late stages of lignification (Fig. 3a). The H/G ratio in developing shoots of $S$. tootsik showed no apparent trend, but remained relatively constant or increased incrementally towards maturity (Fig. 4a). The role of $\mathrm{H}$ units in lignin polymers may differ between grasses and trees.
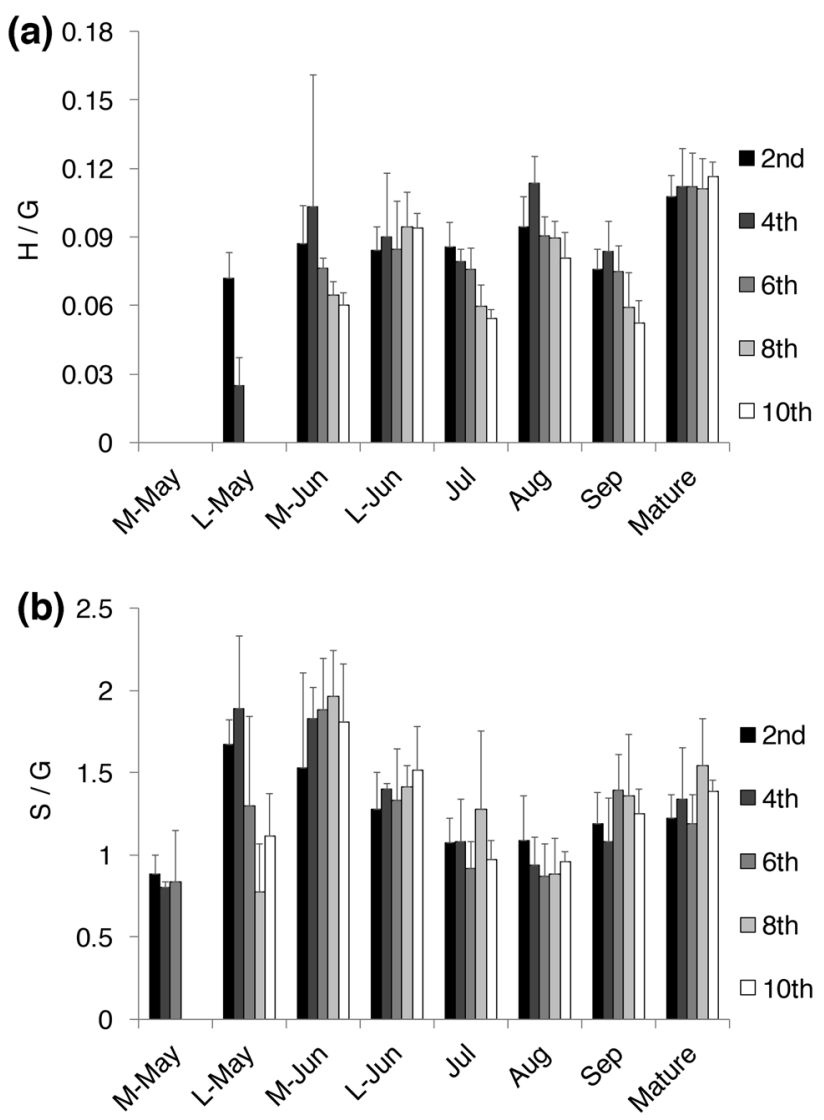

Fig. 4 Ratios of $\mathrm{H} / \mathrm{G}$ and $\mathrm{S} / \mathrm{G}$ units in developing culms of $S$. tootsik. Ratios of a H/G and b S/G units of lignin released by thioacidolysis. $\mathrm{H} / \mathrm{G}$ ratio was not calculated for mid-May sample or 6th, 8th, and 10th internodes of late-May sample, because $\mathrm{H}$ monomers were not detected. See Table 1 for sampling details. Data are mean \pm SD of three biological replicates

In contrast to the $\mathrm{H} / \mathrm{G}$ ratio, the $\mathrm{S} / \mathrm{G}$ ratio varied in developing shoots of $S$. tootsik (Fig. 4b). In the thioacidolysis analysis, the yield of monomer $\mathrm{G}$ units gradually increased from May to August, whereas that of S units increased from May to mid-June, remained stable until late August, and then increased again after August (Fig. 3b, c). Consequently, the $S / G$ ratio tended to increase from midMay to mid-June, and significantly decrease from mid-June to August at all internodes except the 2nd one $(p<0.05$, $t$ test; Fig. $4 \mathrm{~b}$ ). This variation in the $\mathrm{S} / \mathrm{G}$ ratio indicated that the formation rate of $\beta-O-4$ linkages or the transport rate of precursors differed between $\mathrm{G}$ and $\mathrm{S}$ units during the development of $S$. tootsik shoots.

\section{Contents of lignin precursors in developing shoots of $S$. tootsik}

Understanding the occurrence and distribution of lignin precursors will contribute to our knowledge of lignin biosynthesis; however, no studies have investigated these 
topics in bamboo. Here, we determined the distribution of several lignin precursors in developing shoots of $S$. tootsik by LC-MS analyses (Fig. 5). There were larger amounts of monolignols than their corresponding glucosides at their peaks. The monolignols and their glucosides showed different patterns of accumulation. The monolignols contents peaked during late stages of lignification in August (Fig. 5a, c, e), whereas the monolignol glucosides contents peaked during early stages (Fig. 5b, d, f). These results
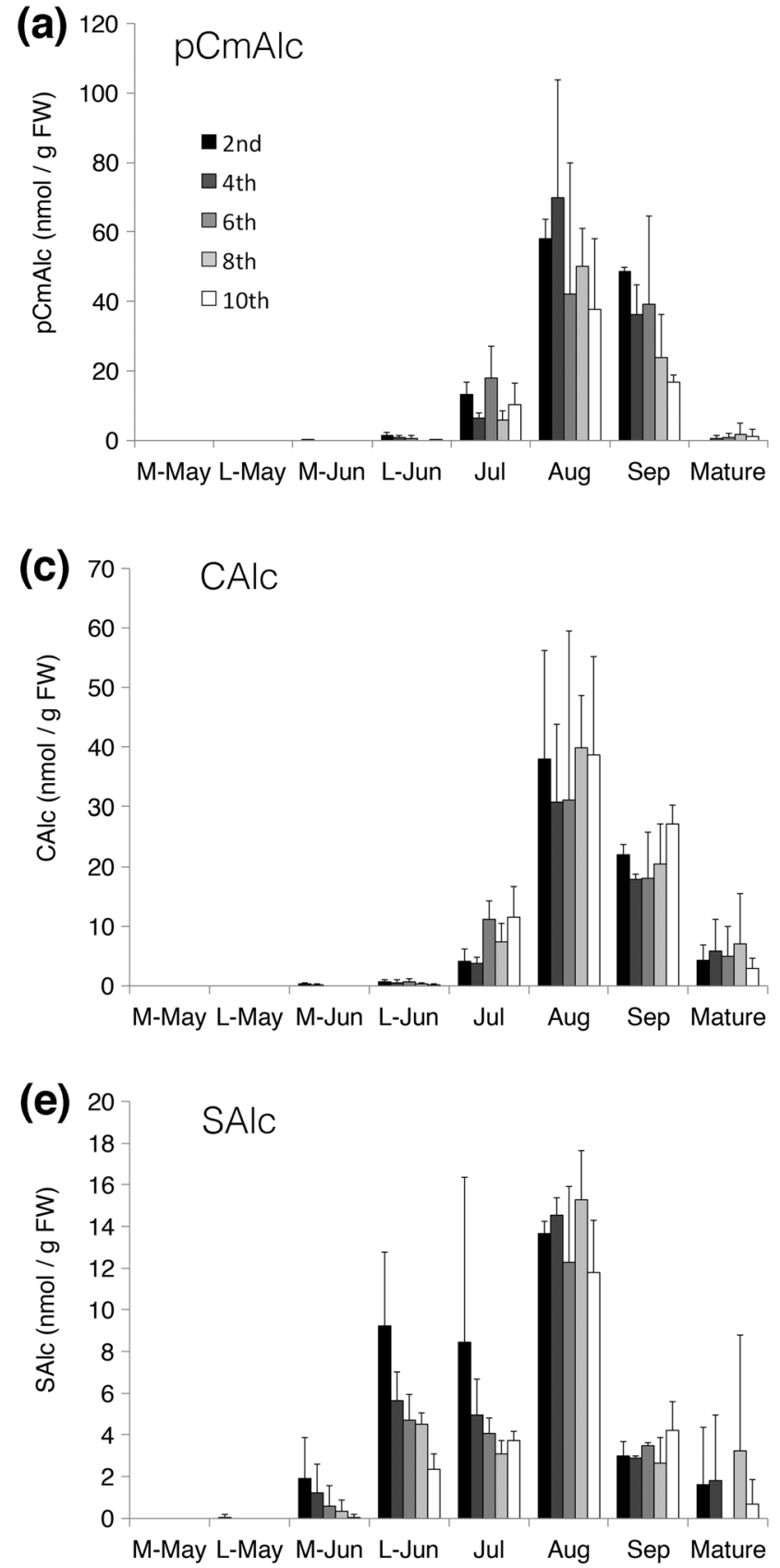

Fig. 5 Distribution of lignin precursors in developing culms of $S$. tootsik. Contents of a p-coumaryl alcohol (pCmAlc), b p-glucocoumaryl alcohol (pCmAlcG), c coniferyl alcohol (CAlc), suggest that lignin precursors may be supplied differentially as lignification proceeds.

Although monolignols are known to be direct precursors of lignin, whether monolignol glucosides are also involved in lignification is controversial. The biosynthesis and hydrolysis of monolignol glucosides have been investigated in several studies [30-35]. A single-gene knockout mutant defective in $\beta$-glucosidase, which is responsible for coniferin and syringin hydrolysis, did not show a
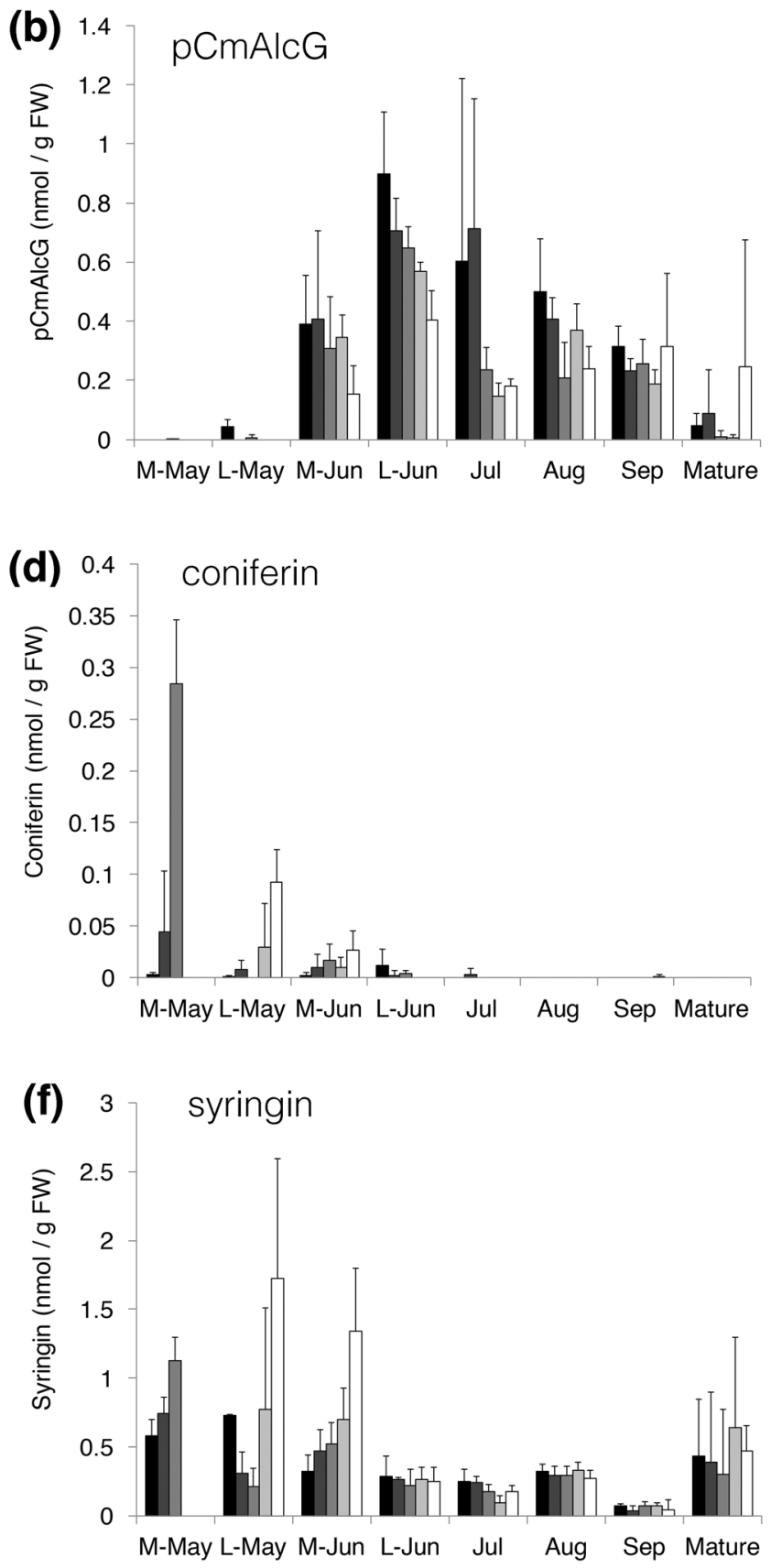

d coniferin, e sinapyl alcohol (SAlc), and $\mathbf{f}$ syringin as determined by LC-MS. See Table 1 for sampling details. Data are mean \pm SD of three biological replicates. $F W$ fresh weight of sample 

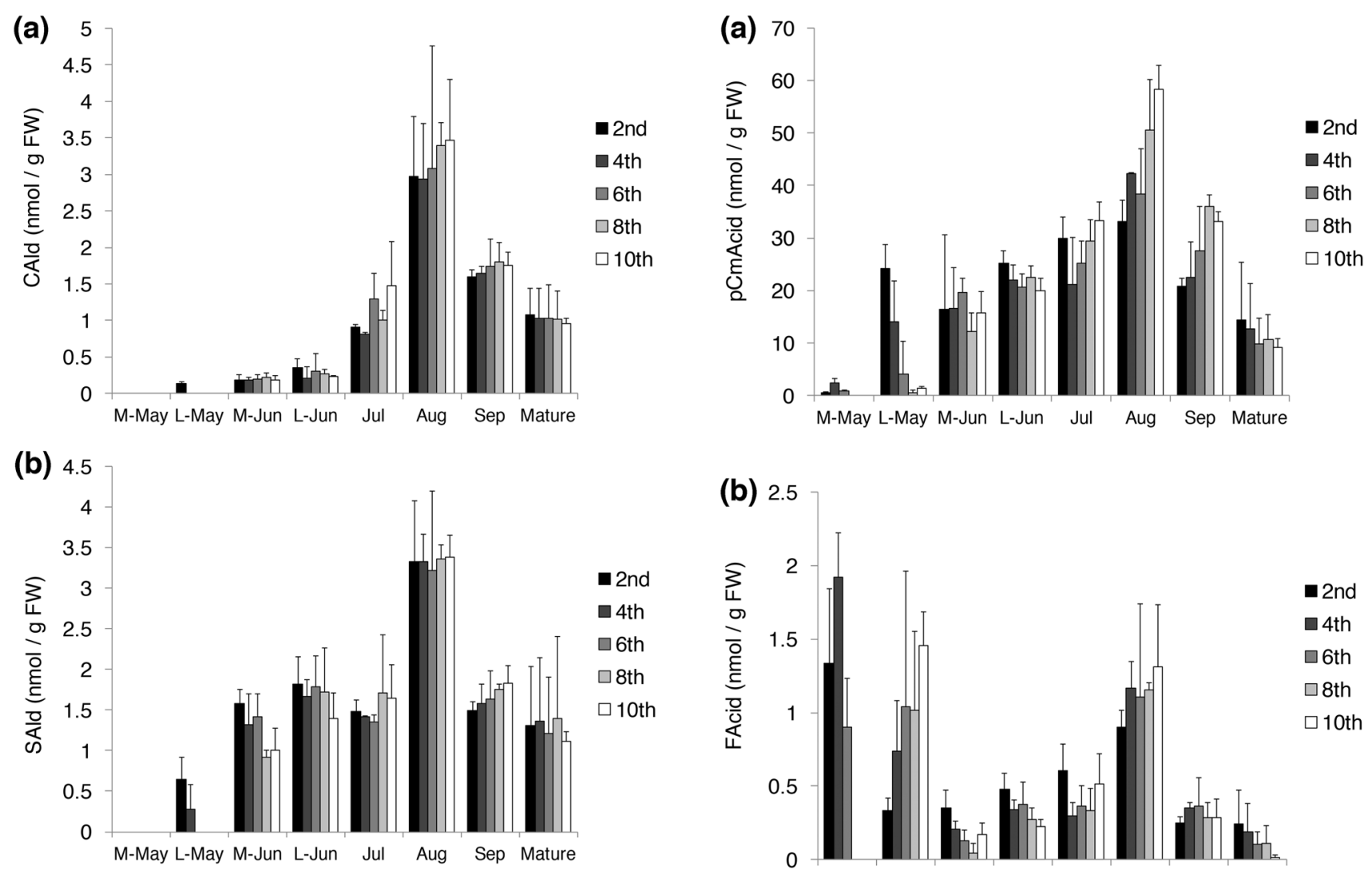

Fig. 6 Distribution of coniferaldehyde and sinapaldehyde in developing culms of $S$. tootsik. Distribution of a coniferaldehyde (CAld) and $\mathbf{b}$ sinapaldehyde (SAld) as determined by LC-MS. See Table 1 for sampling details. Data are mean $\pm \mathrm{SD}$ of three biological replicates. $F W$ fresh weight of sample

lignification-deficient phenotype [36]. However, a recent study revealed that UDP-glycosyltransferase, which is involved in coniferin biosynthesis, is essential for normal lignification in Arabidopsis thaliana [37]. In the present study, coniferin and syringin contents in May and mid-June samples was higher at the upper internodes where vigorous lignification occurs (Fig. 5d, f). The p-glucocoumaryl alcohol content showed a different pattern from those of coniferin or syringin; it peaked in late-June and then decreased as lignification gradually diminished (Fig. 5b). These results suggest that monolignol glucosides are involved in the early stage of lignification in S. tootsik. There were much smaller amounts of monolignol glucosides than monolignols (Fig. 5), implying that there are lower biosynthesis or faster turnover rates of monolignol glucosides. Further research is required to determine the role of monolignol glucosides in bamboo lignification.

Coniferaldehyde and sinapaldehyde were detected in these samples and changes in their contents were almost identical to that of monolignols (Fig. 6). The plasma membranes of rosette leaves of $A$. thaliana showed transport activity not only for monolignols but also for

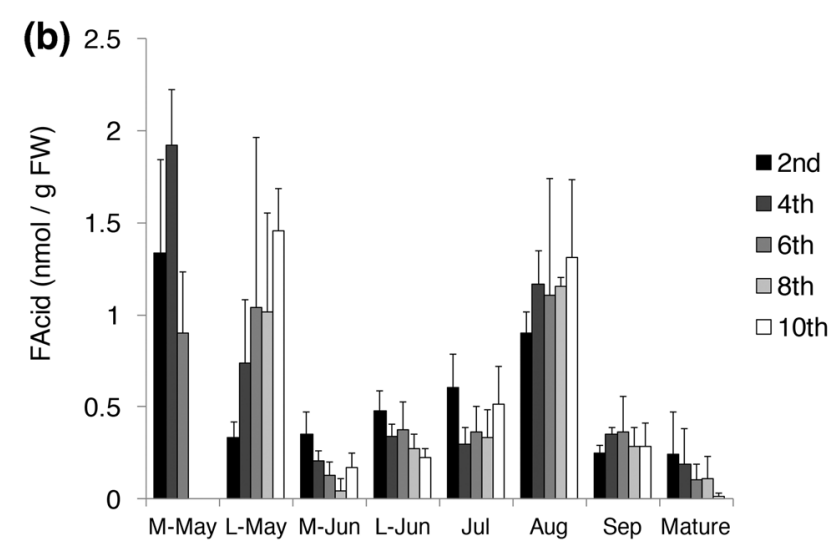

Fig. 7 Distribution of $p$-coumaric acid and ferulic acid in developing culms of $S$. tootsik. Distributions of a $p$-coumaric acid (pCmAcid) and b ferulic acid (FAcid) as determined by LC-MS. See Table 1 for sampling details. Data are mean $\pm \mathrm{SD}$ of three biological replicates. $F W$ fresh weight of sample

coniferaldehyde and sinapaldehyde [12]. Thus, these aldehydes may be transported and used for lignification.

Monocots, including bamboos, contain $p$-coumaric acid and ferulic acid [38]. In late-May, the $p$-coumaric acid content increased from the top to the bottom of the culm (Fig. 7a), whereas the ferulic acid content increased from the bottom to the top of the culm (Fig. 7b). Similar results were reported for shoots of the bamboo $P$. pubescens sampled in May [22]. In grasses, $p$-coumaric acid is mainly incorporated into lignin [38]. The $p$-coumaric acid detected in S. tootsik in late-May appeared to be related to lignification. Ferulic acid is mainly attached to arabinoxylan by an ester linkage that strengthens the cell wall by crosslinking. A portion of the arabinoxylan-attached ferulic acid might function as an initiation site for lignification [38]. The higher content of ferulic acid in elongating internodes (Figs. 1, 7) suggests that ferulic acid plays a role in the early stages of cell wall formation in S. tootsik.

The contents of various lignin precursors, monolignols, $p$-hydroxycinnamaldehydes, $p$-coumaric acid, and ferulic acid peaked in August in $S$. tootsik (Figs. 5, 6, 7). This may 
be a consequence of a delay in the consumption of lignin precursors and the pool of intermediate compounds; indeed, the rate of lignification decreased during August (Fig. 2b). Accumulation of lignin precursors and declining lignification in August may result from diminished transport activity if the precursors are localized in the cytosol, or decreased polymerization activity if the precursors are present in the cell wall. The rate-limiting step for the cessation of lignification in bamboo may not be the biosynthesis of lignin precursors, but rather their transport or polymerization. Further research is needed to elucidate how lignin precursors are transported to the cell wall, and how lignification is controlled during culm development.

\section{Conclusion}

In this study, we evaluated the patterns of culm elongation, lignification, lignin structure, and the distribution of lignin precursors during the development of $S$. tootsik shoots. Elongation growth was completed by late-June when the culm lignin content is approximately half that of mature culms. Thioacidolysis analysis indicated that $\mathrm{H}$ units were almost constantly incorporated into lignin polymers during culm development, whereas the ratio of $\mathrm{S} / \mathrm{G}$ units was variable. The contents of monolignol glucosides peaked during the early stages of lignification, whereas the contents of monolignols peak at later stages of lignification. These details of the variations in lignin structure and the contents of lignin precursors in developing shoots of $S$. tootsik contribute to our understanding of lignification in bamboo, and will help to optimize the use of the bamboo biomass.

Acknowledgements We gratefully acknowledge Dr. Noritsugu Terashima, Nagoya University, for the gift of coniferin and syringin; Dr. Kentaro Sakai and Dr. Tomoko Matsumoto, University of Miyazaki, for technical support with LC-MS analysis; and Dr. Takuya Tetsumura, University of Miyazaki, for generous assistance with cryosectioning.

\section{References}

1. Scurlock JMO, Dayton DC, Hames B (2000) Bamboo: an overlooked biomass resource? Biomass Bioenergy 19:229-244

2. He MX, Wang JL, Qin H, Shui ZX, Zhu QL, Wu B, Tan FR, Pan $\mathrm{K}, \mathrm{Hu}$ QC, Dai LC, Wang WG, Tang XY, Hu GQ (2014) Bamboo: a new source of carbohydrate for biorefinery. Carbohydr Polym 111:645-654

3. Xiao X, Bian J, Li MF, Xu H, Xiao B, Sun RC (2014) Enhanced enzymatic hydrolysis of bamboo (Dendrocalamus giganteus Munro) culm by hydrothermal pretreatment. Bioresour Technol 159:41-47

4. Yang Z, Zhang M, Xin D, Wang J, Zhang J (2014) Evaluation of aqueous ammonia pretreatment for enzymatic hydrolysis of different fractions of bamboo shoot and mature bamboo. Bioresour Technol 173:198-206
5. Jiang Z, Fei B, Li Z (2016) Pretreatment of bamboo by ultra-high pressure explosion with a high-pressure homogenizer for enzymatic hydrolysis and ethanol fermentation. Bioresour Technol 214:876-880

6. Chang VS, Holtzapple MT (2000) Fundamental factors affecting biomass enzymatic reactivity. Appl Biochem Biotechnol 84-86:5-37

7. Öhgren K, Bura R, Saddler J, Zacchi G (2007) Effect of hemicellulose and lignin removal on enzymatic hydrolysis of steam pretreated corn stover. Bioresour Technol 98:2503-2510

8. Shimokawa T, Ishida M, Yoshida S, Nojiri M (2009) Effects of growth stage on enzymatic saccharification and simultaneous saccharification and fermentation of bamboo shoots for bioethanol production. Bioresour Technol 100:6651-6654

9. Higuchi T (1969) Bamboo lignin and its biosynthesis. Wood Res 48:1-14

10. Yoshizawa N, Satoh I, Yokota S, Idei T (1991) Lignification and peroxidase activity in bamboo shoots (Phyllostachys edulis A. et C. Riv.). Holzforschung 45:169-174

11. Vanholme R, Morreel K, Darrah C, Oyarce P, Grabber JH, Ralph J, Boerjan W (2012) Metabolic engineering of novel lignin in biomass crops. New Phytol 196:978-1000

12. Miao YC, Liu CJ (2010) ATP-binding cassette-like transporters are involved in the transport of lignin precursors across plasma and vacuolar membranes. Proc Natl Acad Sci USA 107:22728-22733

13. Alejandro S, Lee Y, Tohge T, Sudre D, Osorio S, Park J, Bovet L, Lee Y, Geldner N, Fernie AR, Martinoia E (2012) AtABCG29 is a monolignol transporter involved in lignin biosynthesis. Curr Biol 22:1207-1212

14. Tsuyama T, Kawai R, Shitan N, Matoh T, Sugiyama J, Yoshinaga A, Takabe K, Fujita M, Yazaki K (2013) Proton-dependent coniferin transport, a common major transport event in differentiating xylem tissue of woody plants. Plant Physiol 162:918-926

15. Fukushima K, Taguchi S, Matsui N, Yasuda S (1997) Distribution and seasonal changes of monolignol glucosides in Pinus thunbergii. Mokuzai Gakkaishi 43:254-259

16. Tsuyama T, Takabe K (2014) Distribution of lignin and lignin precursors in differentiating xylem of Japanese cypress and poplar. J Wood Sci 60:353-361

17. Aoki D, Hanaya Y, Akita T, Matsushita Y, Yoshida M, Kuroda K, Yagami S, Takama R, Fukushima K (2016) Distribution of coniferin in freeze-fixed stem of Ginkgo biloba L. by cryo-TOFSIMS/SEM. Sci Rep 6:31525

18. Fukushima K, Taguchi S, Matsui N, Yasuda S (1996) Heterogeneous distribution of monolignol glucosides in the stems of Magnolia kobus. Mokuzai Gakkaishi 42:1029-1031

19. Parameswaran N, Liese W (1976) On the fine structure of bamboo fibres. Wood Sci Technol 10:231-246

20. Itoh T (1990) Lignification of bamboo (Phyllostachys heterocycla Mitf.) during its growth. Holzforschung 44:191-200

21. Lin J, He X, Hu Y, Kuang T, Ceulemans R (2002) Lignification and lignin heterogeneity for various age classes of bamboo (Phyllostachys pubescens) stems. Physiol Plant 114:296-302

22. Fujii Y, Azuma J, Marchessault RH, Morin FG, Aibara S, Okamura K (1993) Chemical composition change of bamboo accompanying its growth. Holzforschung 47:109-115

23. Terashima N, Ralph SA, Landucci LL (1996) New facile syntheses of monolignol glucosides; $p$-glucocoumaryl alcohol, coniferin, and syringin. Holzforschung 50:151-155

24. Suzuki S, Suzuki Y, Yamamoto N, Hattori T, Sakamoto M, Umezawa T (2009) High-throughput determination of thioglycolic acid lignin from rice. Plant Biotechnol 26:337-340 
25. Yamamura M, Hattori $T$, Suzuki S, Shibata D, Umezawa $T$ (2012) Microscale thioacidolysis method for the rapid analysis of $\beta-O-4$ substructures in lignin. Plant Biotechnol 29:419-423

26. Okamura H, Tanaka Y, Konishi M, Kashiwagi H (eds) (1991) Illustrated horticultural bamboo species in Japan. Haato, Tokyo

27. Liese W, Weiner G (1996) Ageing of bamboo culms. A review. Wood Sci Technol 30:77-89

28. Terashima N, Fukushima K (1988) Heterogeneity in formation of lignin-XI: an autoradiographic study of the heterogeneous formation and structure of pine lignin. Wood Sci Technol 22:259-270

29. Terashima N, Fukushima K, Takabe K (1986) Heterogeneity in formation of lignin. VIII. An autoradiographic study on the formation of guaiacyl and syringyl lignin in Magnolia kobus DC. Holzforschung 40(Suppl):101-105

30. Dharmawardhana DP, Ellis BE, Carlson JE (1995) A $\beta$-glucosidase from lodgepole pine xylem specific for the lignin precursor coniferin. Plant Physiol 107:331-339

31. Savidge RA, Förster H (1998) Seasonal activity of uridine $5^{\prime}$ diphosphoglucose:coniferyl alcohol glucosyltransferase in relation to cambial growth and dormancy in conifers. Can $\mathrm{J}$ Bot 76:486-493

32. Lanot A, Hodge D, Jackson RG, George GL, Elias L, Lim EK, Vaistij FE, Bowles DJ (2006) The glucosyltransferase UGT72E2 is responsible for monolignol 4-O-glucoside production in Arabidopsis thaliana. Plant J 48:286-295
33. Escamilla-Treviño LL, Chen W, Card ML, Shih MC, Cheng CL, Poulton JE (2006) Arabidopsis thaliana $\beta$-glucosidases BGLU45 and BGLU46 hydrolyse monolignol glucosides. Phytochemistry 67:1651-1660

34. Tsuyama T, Takabe $\mathrm{K}$ (2015) Coniferin $\beta$-glucosidase is ionically bound to cell wall in differentiating xylem of poplar. J Wood Sci $61: 438-444$

35. Terashima N, Ko C, Matsushita Y, Westermark U (2016) Monolignol glucosides as intermediate compounds in lignin biosynthesis. Revisiting the cell wall lignification and new ${ }^{13} \mathrm{C}$ tracer experiments with Ginkgo biloba and Magnolia liliiflora. Holzforschung 70:801-810

36. Chapelle A, Morreel K, Vanholme R, Le-Bris P, Morin H, Lapierre C, Boerjan W, Jouanin L, Demont-Caulet N (2012) Impact of the absence of stem-specific $\beta$-glucosidases on lignin and monolignols. Plant Physiol 160:1204-1217

37. Lin JS, Huang XX, Li Q, Cao Y, Bao Y, Meng XF, Li YJ, Fu C, Hou BK (2016) UDP-glycosyltransferase $72 \mathrm{~B} 1$ catalyzes the glucose conjugation of monolignols and is essential for the normal cell wall lignification in Arabidopsis thaliana. Plant $\mathrm{J}$ 88:26-42

38. Hatfield RD, Marita JM (2010) Enzymatic processes involved in the incorporation of hydroxycinnamates into grass cell walls. Phytochem Rev 9:35-45 